\title{
A simulation study of the decay of the pair correlation function in simple fluids
}

\author{
Marjolein Dijkstra ${ }^{a)}$ and Robert Evans \\ H. H. Wills Physics Laboratory, University of Bristol, Royal Fort, Bristol BS8 1TL, United Kingdom
}

(Received 23 August 1999; accepted 21 October 1999)

\begin{abstract}
Monte Carlo simulations are used to investigate the asymptotic decay of the total pairwise correlation function $h(r)$ for some model fluids. We determine the poles of the Fourier transform $\hat{h}(q)$ from the direct correlation function $c(r)$. The leading poles determine the ultimate, $r \rightarrow \infty$, decay of $h(r)$. For the truncated and shifted Lennard-Jones fluid we calculate the Fisher-Widom (disorder) line in the temperature-density $(T, \rho)$ plane where the ultimate decay of $r h(r)$ crosses over from monotonic (exponential) to exponentially damped oscillatory decay. This line lies close to that obtained in an earlier integral-equation [hypernetted chain-soft core mean spherical approximation (HMSA)] study. For states on the monotonic side of the disorder line, $h(r)$ has a finite number of oscillations and we determine the boundaries which mark regions in the $(T, \rho)$ plane where $h(r)$ has a given number of zeros using a random-phase approximation for $c(r)$. In the case of the hard-sphere fluid, the ultimate decay of $h(r)$ is oscillatory for all densities and we find that simulation results for the period and (exponential) decay length of the oscillations are in good overall agreement with those of Percus-Yevick theory, although there is some indication that systematic differences develop for high-density states $\rho^{*} \geqslant 0.85$. (C) 2000 American Institute of Physics. [S0021-9606(00)51003-4]
\end{abstract}

\section{INTRODUCTION}

It is now 30 years since Fisher and Widom ${ }^{1}$ conjectured, on the basis of some exact results for one-dimensional models, that there should be a line in the temperature-density $(T, \rho)$ plane where the longest range decay of the total pairwise correlation function $h(r)$ of a simple fluid should cross over from monotonic to exponentially damped oscillatory. For high temperatures and densities, repulsive forces and packing considerations dominate and $h(r)$ should exhibit oscillatory decay. At lower densities and temperatures, attractive interatomic forces play a more important role and $h(r)$ should decay in a monotonic fashion, as in Ornstein-Zernike theory. In magnetism, spin-spin correlations can also exhibit different types of asymptotic decay and the analog of the cross over or Fisher-Widom (FW) line is well-known and is termed the disorder transition line. For bulk fluids and magnets, the change in asymptotic decay of $h(r)$ which occurs at the crossover line does not imply any thermodynamic singularity and little attention was paid to the crossover phenomenon until Evans $e t a l .{ }^{2}$ pointed out its repercussions for the structure of fluid interfaces. The density profile of a fluid far from a planar wall exhibits the same type of decay as the bulk $h(r)$. Thus, if $r h(r) \sim \exp \left(-\alpha_{0} r\right)$ for $r \rightarrow \infty$, then the density profile at a wall (exerting a short-ranged wall-fluid potential) will decay as $\exp \left(-\alpha_{0} z\right)$, where $z$ is the distance from the wall, whereas if $r h(r) \sim \exp \left(-\tilde{\alpha}_{0} r\right) \cos \left(\tilde{\alpha}_{1} r-\theta\right)$ then the profile decays as $\exp \left(-\tilde{\alpha}_{0} z\right) \cos \left(\tilde{\alpha}_{1} z-\theta_{w}\right){ }^{2,3}$ This observation has significant implications for the structure of the liquid-vapor interface ${ }^{2}$ and for the occurrence of wetting

\footnotetext{
a) Present address: Debye Institute, Condensed Matter Physics, Utrecht University, Postbus 80000,3508 TA Utrecht, The Netherlands.
}

transitions at wall-fluid interfaces. ${ }^{2,4}$ The first attempt at calculating the FW line for a realistic model of a fluid was based on the random phase approximation (RPA) for the square-well model. The FW line, which is the line in the $(T, \rho)$ plane where $\alpha_{0}=\tilde{\alpha}_{0}$, was found to intersect the liquid branch of the liquid-gas coexistence curve at $T / T_{c} \sim 0.9$ and $\rho / \rho_{c} \sim 1.9$, where $T_{c}$ and $\rho_{c}$ are the critical temperature and density. ${ }^{2}$ Later, the accurate HMSA integral equation theory was used to determine the FW line for the truncated and shifted (at $R_{c}=2.5 \sigma$ ) Lennard-Jones fluid. ${ }^{5}$ The calculated FW line intersected the coexistence curve at values of $T / T_{c}$ and $\rho / \rho_{c}$ close to those stated above (see also Fig. 4). Subsequent investigations of asymptotic decay and crossover lines have focused on Coulombic or screened Coulombic fluids $^{6,7}$ where the crossover mechanisms associated with charge correlations are different from those in simple, atomic fluids described by short-ranged interatomic potentials.

All the studies of the asymptotic decay of correlations reported hitherto have employed approximate theories of liquids, with varying degrees of sophistication. In the present paper we present the first study of the asymptotic decay using computer simulations. At first sight this might appear to be an especially difficult task-measuring $h(r)$ directly at large interatomic separation $r$ requires very large system sizes and good statistics. However, we show that by using the Ornstein-Zernike equation to calculate the direct correlation function $c(r)$ it is possible to extract the longest range decay of $h(r)$ and determine the FW line without recourse to simulations with enormous numbers of particles. We restrict attention to model fluids with interatomic potentials that are of finite range. For fluids in which the pairwise potential $\phi(r)$ decays as a power law, $h(r)$ decays ultimately as $-S^{2}(0) \phi(r) / k_{B} T$, where $S(0)$ is the $q \rightarrow 0$ limit of the liq- 
uid structure factor, ${ }^{8,9}$ and there is no sharp FW line, although there is still erosion of the intermediate range oscillations of $h(r)$ as the density $\rho$ is decreased. ${ }^{5}$

Our paper is arranged as follows: in Sec. II we summarize the key results for the asymptotics of correlation functions and describe the theoretical framework for calculating the inverse decay lengths $\alpha_{0}, \tilde{\alpha}_{0}$ and the period $2 \pi / \tilde{\alpha}_{1}$ which describe the decay of $h(r)$-see above. Our Monte Carlo simulation studies of the Lennard-Jones fluid, truncated and shifted at $R_{c}=2.5 \sigma$, are reported in Sec. III. We measure $h(r)$, extract $c(r)$, and determine the quantities $\alpha_{0}, \tilde{\alpha}_{0}$, and $\tilde{\alpha}_{1}$ from the latter. The FW line which we obtain lies very close to that obtained in Ref. 5 from the HMSA for the same potential. Moreover, we find that leading order asymptotics provide an accurate fit to the simulation results for $h(r)$ at intermediate separations, i.e., for $r$ as small as $2 \sigma$, as well as at long range. In Sec. IV we focus on the hardsphere fluid, where the decay of $h(r)$ is always oscillatory, and determine the decay length and period using simulation results for $c(r)$. The results are compared with those from Percus-Yevick theory. Section V addresses the issue of how the FW line behaves at very high temperatures where the effects of attraction become weak and where the decay of $h(r)$ should become hard-sphere-like. Section VI describes an RPA analysis of the number of zeros of $h(r)$ for states on the monotonic side of the FW line. We determine boundaries in the $(T, \rho)$ plane which separate a region where $h(r)$ has $n$ zeros from one with $n+2$ zeros. For states approaching the FW line, $n$ increases to infinity and the boundaries cluster. Finally, in Sec. VII we make some concluding remarks.

\section{THEORY}

Below we give a brief summary of the asymptotics of the pair correlation function of a fluid with short-ranged interatomic potentials. For more details we refer the reader to Refs. 2 and 3. The asymptotic decay of the radial distribution function $g(r)$ is most easily determined by investigating the pole structure of the structure factor $S(q)$. The OrnsteinZernike (OZ) equation relates the total pair correlation function $h(r)=g(r)-1$ to the direct correlation function $c(r)$

$$
h(r)=c(r)+\rho \int d \mathbf{r}^{\prime} h\left(r^{\prime}\right) c\left(\left|\mathbf{r}-\mathbf{r}^{\prime}\right|\right),
$$

where $\rho$ denotes the bulk density. In Fourier representation, this equation reads

$$
\hat{h}(q)=\frac{\hat{c}(q)}{1-\rho \hat{c}(q)},
$$

where the Fourier transform $\hat{f}(q)$ of a spherically symmetric function $f(r)$ is given by

$$
\hat{f}(q)=4 \pi \int_{0}^{\infty} d r r^{2} f(r) \frac{\sin q r}{q r},
$$

or inversely by

$$
f(r)=\frac{1}{2 \pi^{2}} \int_{0}^{\infty} d q q^{2} \hat{f}(q) \frac{\sin q r}{q r} .
$$

Combining Eqs. (2) and (4) yields

$$
r h(r)=\frac{1}{2 \pi^{2}} \int_{0}^{\infty} d q q \sin q r \frac{\hat{c}(q)}{1-\hat{\rho}(q)} .
$$

For short-ranged interatomic potentials, where $c(r)$ decays faster than a power law, it follows that the asymptotic behavior of $r h(r)$, i.e., $r \rightarrow \infty$, is determined by the poles of $\hat{h}(q)$, i.e., poles at complex $q=\alpha \equiv \alpha_{1}+i \alpha_{0}$ that satisfy

$$
1-\rho \hat{c}(\alpha)=0
$$

A pole can lie on the imaginary axis where it gives rise to pure exponential decay of $r h(r)$, or it can lie off the imaginary axis where it gives rise to exponentially damped oscillatory decay. Equating the real and imaginary parts in Eq. (6) gives

$$
\begin{aligned}
& 1=4 \pi \rho \int_{0}^{\infty} d r r^{2} c(r) \frac{\sinh \left(\alpha_{0} r\right)}{\alpha_{0} r} \cos \left(\alpha_{1} r\right), \\
& 1=4 \pi \rho \int_{0}^{\infty} d r r^{2} c(r) \cosh \left(\alpha_{0} r\right) \frac{\sin \left(\alpha_{1} r\right)}{\alpha_{1} r} .
\end{aligned}
$$

Provided $c(r)$ is known for a given density and temperature, this pair of equations can be used to find the poles $q=\alpha_{1}$ $+i \alpha_{0}$. A pure imaginary pole is obtained from Eq. (7) alone with $\alpha_{1}=0$. The right-hand side of Eq. (5) can be evaluated by choosing the contour to be an infinite semicircle in the upper half-plane, and provided all poles are simple it follows that

$$
r h(r)=\sum_{n} e^{i q_{n} r} A_{n} \text { with } A_{n}=-\frac{q_{n}}{2 \pi \rho^{2} \hat{c}^{\prime}\left(q_{n}\right)},
$$

where $q_{n}$ is the $n$-th pole, $2 \pi A_{n}$ is the residue of $q \hat{c}(q) /(1$ $-\hat{\rho}(q))$ at $q=q_{n}$, and $\hat{c}^{\prime}\left(q_{n}\right)$ is the derivative of $\hat{c}(q)$ with respect to $q$ at $q=q_{n}$. In general, an infinite number of poles can be expected ${ }^{10}$ but the longest range part of $h(r)$ is determined by the pole or poles with the smallest imaginary part. Two scenarios are possible: (a) Pure exponential decay dominates at longest range if the pole lying on the imaginary axis $q=i \alpha_{0}$ has the smallest value of $\alpha_{0}$. Using Eq. (9), we find that the contribution of a pure imaginary pole to $r h(r)$ is given by

$$
r h(r) \sim A e^{-\alpha_{0} r}
$$

with an amplitude

$$
A=-\frac{i \alpha_{0}}{2 \pi \rho^{2} \hat{c}^{\prime}\left(i \alpha_{0}\right)}
$$

and

$\hat{c}^{\prime}\left(i \alpha_{0}\right)=\frac{4 \pi}{i \alpha_{0}} \int_{0}^{\infty} d r r^{2} c(r)\left[\cosh \left(\alpha_{0} r\right)-\frac{\sinh \left(\alpha_{0} r\right)}{\alpha_{0} r}\right]$.

(b) The complex poles in the upper half-plane occur as conjugate pairs and exponentially damped oscillatory decay with a wavelength $2 \pi / \tilde{\alpha}_{1}$ dominates at longest range, if a conjugate pair of poles $q= \pm \tilde{\alpha}_{1}+i \tilde{\alpha}_{0}$ has a smaller imaginary 
part than the pole on the imaginary axis. The decay of $h(r)$ at longest range is then determined by the contribution of the conjugate pair of complex poles, i.e.,

$$
r h(r) \sim 2|\widetilde{A}| e^{-\tilde{\alpha}_{0} r} \cos \left(\tilde{\alpha}_{1} r-\theta\right) .
$$

Explicit formulas for the amplitude and phase are derived in Refs. 3 and 6 and read

$$
|\widetilde{A}| e^{-i \theta}=\frac{\left(\tilde{\alpha}_{0}^{2}+\widetilde{\alpha}_{1}^{2}\right)}{8 \pi^{2} \rho^{2} \sqrt{\tilde{a}^{2}+\tilde{b}^{2}}} e^{-i(t-2 p)}, \text { and }
$$

with the angles $p$ and $t$ determined by

$$
e^{i p}=\frac{\tilde{\alpha}_{1}+i \tilde{\alpha}_{0}}{\sqrt{\tilde{\alpha}_{0}^{2}+\tilde{\alpha}_{1}^{2}}}, \quad e^{i t}=\frac{\tilde{a}+i \tilde{b}}{\sqrt{\tilde{a}^{2}+\tilde{b}^{2}}},
$$

and

$$
\tilde{a}=\frac{a \tilde{\alpha}_{0}+b \tilde{\alpha}_{1}}{\tilde{\alpha}_{0}^{2}+\tilde{\alpha}_{1}^{2}}-c, \quad \tilde{b}=\frac{a \tilde{\alpha}_{1}-b \tilde{\alpha}_{0}}{\tilde{\alpha}_{0}^{2}+\tilde{\alpha}_{1}^{2}}+d,
$$

where the functions $a, b, c$, and $d$ are defined by

$$
\begin{aligned}
& a=\int_{0}^{\infty} d r r c(r) \sinh \left(\tilde{\alpha}_{0} r\right) \cos \left(\tilde{\alpha}_{1} r\right), \\
& b=\int_{0}^{\infty} d r r c(r) \cosh \left(\tilde{\alpha}_{0} r\right) \sin \left(\tilde{\alpha}_{1} r\right), \\
& c=\int_{0}^{\infty} d r r^{2} c(r) \cosh \left(\tilde{\alpha}_{0} r\right) \cos \left(\tilde{\alpha}_{1} r\right), \\
& d=\int_{0}^{\infty} d r r^{2} c(r) \sinh \left(\tilde{\alpha}_{0} r\right) \sin \left(\tilde{\alpha}_{1} r\right) .
\end{aligned}
$$

The crossover from monotonic decay to damped oscillatory decay at longest range is given by the so-called FisherWidom $(\mathrm{FW})$ line. The locus of the FW line in the $(\rho, T)$ plane is determined by the condition $\alpha_{0}=\tilde{\alpha}_{0}$. In order to utilize the procedure described above, all the relevant integrals must converge. This is guaranteed when $c(r)$ is of finite range, as is the case for interatomic potentials $\phi(r)$ of finite range treated in the mean-spherical (MSA) or randomphase (RPA) approximation. In reality, however, $c(r)$ will have a nonvanishing tail for $r>R_{c}$, the cutoff distance. Integral equation theories predict that $c(r)$ decays as

$$
c(r) \sim-\beta \phi(r)+\frac{\tau(r)}{2} h^{2}(r)+\cdots,
$$

where $\beta=1 / k_{B} T$ and $\tau(r)$ is some (slowly varying) function of $r$. Within the hypernetted chain (HNC) approximation $\tau(r) \equiv 1$ while in the HMSA ${ }^{11} 0<\tau(r)<1$. This implies that the integrals in Eqs. (7) and (8) will diverge for any pole whose imaginary part is larger than twice the imaginary part ( $\alpha_{0}$ or $\tilde{\alpha}_{0}$ ) of the leading, i.e., the lowest-lying pole. ${ }^{6}$ Thus, our numerical procedure for determining poles and residues is restricted to the calculation of the two leading poles. Note also that certain integral equation theories yield other types of singularities in $\hat{h}(q)$ which give rise to additional terms in

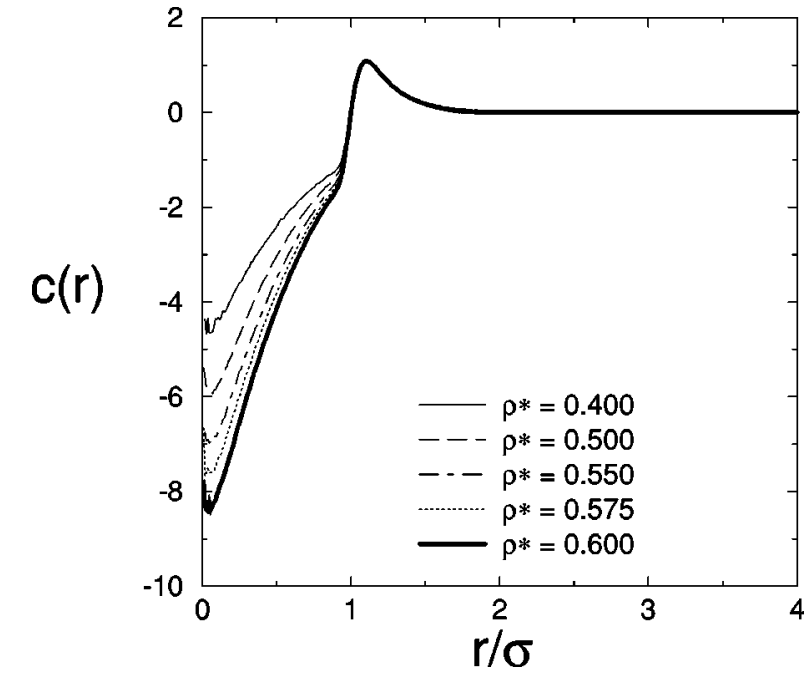

FIG. 1. Monte Carlo results for the direct correlation function $c(r)$ of the truncated and shifted $\mathrm{LJ}$ fluid at reduced temperature $T^{*}=1.25$ for several densities. The oscillations for $r / \sigma \leqslant 0.1$ arise from truncating the Fourier transforms.

Eq. (9). Following earlier work, ${ }^{6}$ we shall assume any such terms decay faster than the contributions of the two leading poles.

\section{MONTE CARLO SIMULATION STUDIES OF THE LENNARD-JONES FLUID}

\section{A. Determination of the Fisher-Widom line}

We performed canonical Monte Carlo simulations of a fluid interacting with a Lennard-Jones potential which is truncated and shifted at $R_{c}=2.5 \sigma$,

$$
\begin{aligned}
\phi_{\mathrm{LJ} t s}(r) & =\phi_{\mathrm{LJ}}(r)-\phi_{\mathrm{LJ}}\left(R_{c}\right) \quad r \leqslant R_{c}, \\
& =0 \quad r>R_{c},
\end{aligned}
$$

with $\phi_{\mathrm{LJ}}(r)=4 \epsilon\left((\sigma / r)^{12}-(\sigma / r)^{6}\right)$. The Gibbs ensemble simulation study of $\mathrm{Smit}^{13}$ estimated the critical point of this model to lie at $T_{c}^{*}=1.085$ and $\rho_{c}^{*}=0.32$. We measured the pair correlation function $g(r)$ at equidistant intervals of $\sigma / 100$ for several reduced temperatures $T^{*}=(\beta \epsilon)^{-1}$ and reduced densities $\rho^{*}=N \sigma^{3} / V$, where $N$ is the number of particles and $V$ the volume of the system. The system consists of a cubic box with length $L=20 \sigma$ containing $N$ particles, where $N$ is the nearest integer to $\rho L^{3}$. We determined the poles by solving the two Eqs. (7) and (8). These require $c(r)$ as input. First, we compute $\hat{h}(q)$ by a Fourier transform of $h(r)=g(r)-1$. We then obtain $\hat{c}(q)$ using the OrnsteinZernike (OZ) equation in Fourier space [Eq. (2)]. The direct correlation function in real space $c(r)$ is obtained from an inverse Fourier transform of $\hat{c}(q)$. Figure 1 shows the direct correlation function $c(r)$ for $T^{*}=1.25$ and $\rho^{*}$ $=0.40,0.50,0.55,0.575$, and 0.60 . We find that the $c(r)$ is negative inside the core, $r<\sigma$, and is positive outside the core, decaying smoothly to zero at large distances. Upon increasing the density, $c(r)$ becomes more negative inside the core as the packing increases and the effects of repulsion become more important. Significantly, for $r$ outside the core, 


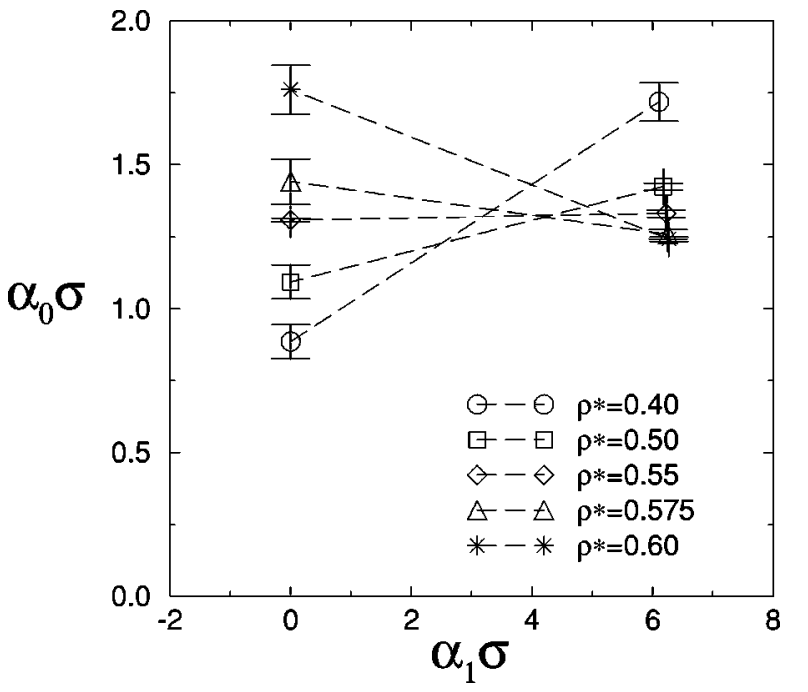

FIG. 2. The imaginary $\left(\alpha_{0}\right)$ and real $\left(\alpha_{1}\right)$ part of the leading poles for several densities as obtained from the results shown in Fig. 1, i.e., for $T^{*}$ $=1.25$. The symbols with $\alpha_{1} \equiv 0$ refer to the pure imaginary pole while those with $\alpha_{1} \sigma \sim 6.1$ refer to the lowest-lying complex pair $\alpha= \pm \tilde{\alpha}_{1}+i \tilde{\alpha}_{0}$ (only the pole with $\tilde{\alpha}_{1}>0$ is shown). The dashed lines couple poles at the same density.

$c(r)$ is almost independent of $\rho^{*}$. Using these $c(r)$ 's, we obtain the leading poles of $h(\hat{q})$ by solving Eqs. (7) and (8). In the first instance we simply truncated the integrals at $2.5 \sigma$, the cutoff value of the potential, since $c(r)$ is very small at this distance. In Fig. 2, we show for each density the pure imaginary pole and the pole off the imaginary axis with the smallest value of $\tilde{\alpha}_{0}$. The statistical error bars (one standard deviation) were estimated by calculating the poles from 3-5 independent sets of $g(r)$ 's. We also performed simulations for $L=25 \sigma$ in order to check the system size dependence and found that the values of these poles agree with those obtained from the smaller system size within the statistical error. Note that the spurious oscillations in $c(r)$ at $r / \sigma$ $\leqslant 0.1$ arise from truncation effects in the Fourier transform. ${ }^{12}$ These do not have a significant effect on the values obtained for the poles since for these values of $r$ the integrands in (7) and (8) vary as $r^{2} c(r)$, which is very small. What is more significant is the large $r$ behavior of $c(r)$ since at large $r$ the integrands vary as $r c(r) \exp \left(\alpha_{0} r\right)$ and, as pointed out in Sec. II, $c(r)$ is not identically zero beyond the range of the potential. In order to check the sensitivity of our results to the tail of $c(r)$ we assumed that $c(r) \sim h^{2}(r) / 2$ for $r>2.5 \sigma$ and calculated $h(r)$ from the two-pole asymptotic formula

$$
r h(r) \sim A e^{-\alpha_{0} r}+2|\widetilde{A}| e^{-\tilde{\alpha}_{0} r} \cos \left(\tilde{\alpha}_{1} r-\theta\right),
$$

i.e., from the pure imaginary plus the lowest-lying conjugate pair of complex poles, using the values of the poles and the residues obtained from the original calculations which have $c(r)=0$ for $r>2.5 \sigma$. Recalculating the poles from (7) and (8) with this form of tail for $c(r)$ changes their values by a small amount. For the lowest density $\rho^{*}=0.4, \alpha_{0}$ changes by $0.26 \%$ and $\tilde{\alpha}_{0}$ by $0.10 \%$, whereas for the highest density, $\rho^{*}=0.6$, the corresponding changes are $4 \%$ and $0.3 \% . \tilde{\alpha}_{1}$ is

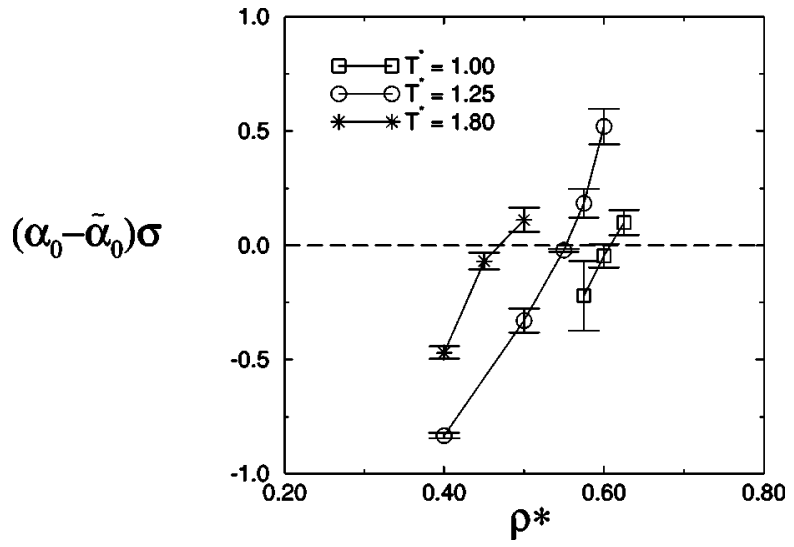

FIG. 3. Monte Carlo results for the difference between the pure imaginary pole $\left(\alpha_{0}\right)$ and the imaginary part of the lowest-lying complex pole $\left(\tilde{\alpha}_{0}\right)$ as a function of reduced density $\rho^{*}$ for three different temperatures. When $\left(\alpha_{0}-\tilde{\alpha}_{0}\right) \sigma<0$ the ultimate decay of $h(r)$ is monotonic whereas for $\left(\alpha_{0}\right.$ $\left.-\tilde{\alpha}_{0}\right) \sigma>0$ it is damped oscillatory.

insensitive to the addition of the tail. Thus, we believe that our results for the two leading poles should not depend very strongly on tail contributions to $c(r)$.

In Fig. 2 we observe that at $\rho^{*}=0.40$ and 0.50 the pure imaginary pole has a smaller imaginary part than the corresponding complex pole, whereas at $\rho^{*}=0.575$ and 0.60 the complex pole has the smaller imaginary part. This indicates that for $T^{*}=1.25$ the decay of $r h(r)$ changes from purely exponential, at longest range, to damped oscillatory as $\rho^{*}$ is increased from 0.50 to 0.575 . Figure 3 displays the difference between $\alpha_{0}$ (for the pure imaginary pole) and $\tilde{\alpha}_{0}$ (for the first complex pole) as a function of $\rho^{*}$. Fitting the data to a cubic spline we estimate the crossover from monotonic decay to damped oscillatory, where $\alpha_{0}=\tilde{\alpha}_{0}$, to occur at $\rho^{*}$ $=0.553$ for $T^{*}=1.25$.

Further sets of calculations were carried out for the subcritical temperature $T^{*}=1.0$ and for $T^{*}=1.8$, and the corresponding results for $\alpha_{0}-\tilde{\alpha}_{0}$ are also plotted in Fig. 3. From these results we have constructed a portion of the FW line in the $\left(T^{*}, \rho^{*}\right)$ plane and this is shown in Fig. 4 along with the FW line obtained from the HMSA integral equation theory. ${ }^{5}$ The two lines have very similar shape and lie close together. The simulation FW line intersects the liquid branch of the coexistence curve of $\mathrm{Smit}^{13}$ at $T^{*} \simeq 0.95$, i.e., at $T / T_{c}$ $=0.88$ and $\rho / \rho_{c}=1.94$.

\section{B. Long and intermediate range decay of $h(r)$}

Integral equation studies of the asymptotic decay of $h(r)$ for a variety of fluids have shown that retaining only the two leading pole contributions in the expansion of (9) leads to a remarkably accurate description of $h(r)$ at intermediate as well as long range. ${ }^{3,5-7}$ More specifically, it was found that the approximation (23) is in very close agreement with the results for $h(r)$ obtained from the direct numerical solution of the integral equations even for interatomic separations as small as $2 \sigma$, i.e., second nearest neighbor distances. It is of 


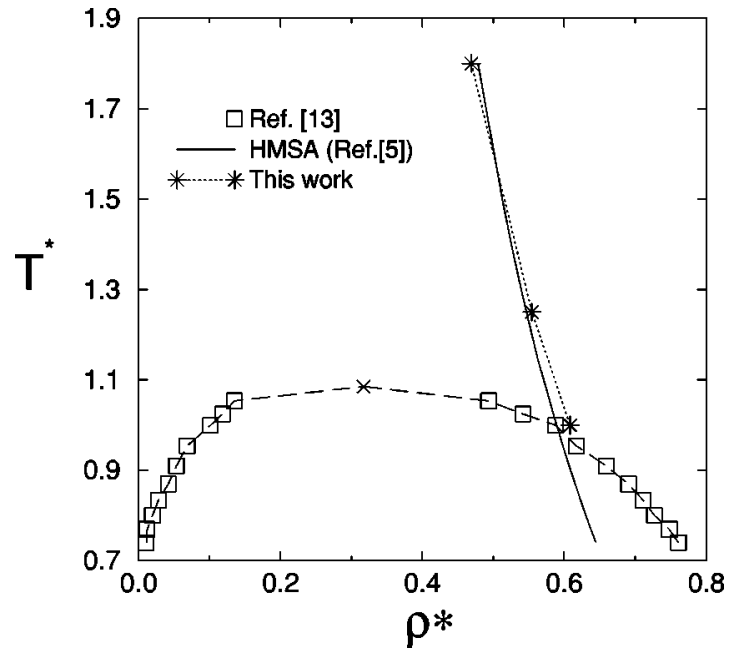

FIG. 4. The Fisher-Widom (FW) line in the temperature-density plane for the LJ fluid truncated and shifted at $2.5 \sigma$. The asterisks joined by dots denote our present Monte-Carlo results for the FW line while the solid curve is the result of the HMSA (Ref. 5). The dashed line joining squares denotes the simulation results (Ref. 13) for the liquid-gas coexistence curve and the cross is the simulation estimate of the critical point.

considerable interest to enquire whether the same approximation provides an equally accurate description of the simulation results.

In Fig. 5(a) we compare the simulation result for $h(r)$ with that from (23) for $T^{*}=1.25$ and $\rho^{*}=0.4$. The poles and the residues, which determine via Eqs. (10)-(20) the amplitudes and the phase, were obtained from $c(r)$ calculated as described above. For this state point $\alpha_{0}<\tilde{\alpha}_{0}$ (see Fig. 2), so the ultimate asymptotic decay of $h(r)$ is predicted to be monotonic. However, the contribution from the conjugate pair of complex poles dominates at intermediate and short range and accounts for the three maxima of $h(r)$ that are easily discernible in the simulations. Equation (23) provides an excellent fit to the simulation result down to $r$ $\sim 2 \sigma$ and it even gives the correct position of the first peak in $h(r)$. The overall quality of the approximation is similar to that found in the HMSA calculations. ${ }^{5}$ Figure 5(b) displays the corresponding results for the same temperature but a higher density, $\rho^{*}=0.6$. Now $\alpha_{0}>\tilde{\alpha}_{0}$ since we have crossed the FW line and the ultimate decay should be damped oscillatory. Once again (23) provides an excellent fit to simulation down to $r \sim 2 \sigma$ and yields the correct first peak position.

The present two-pole approximation appears to be just as reliable at intermediate range as in the earlier integral equation studies, where its success was attributed ${ }^{5-7}$ to the fact that the next lowest-lying complex poles have much larger imaginary parts. Thus we conclude that were we able to calculate the next poles by simulation these would also be far removed from the lowest-lying ones.

As a remark on the usefulness of our asymptotic analysis we should note that naive comparison of the simulation results for $h(r)$ in Figs. 5(a) and 5(b) would not establish a conclusive difference between the two forms of asymptotic decay. Although one might ascertain one or two more oscillations for $\rho^{*}=0.6$ than for $\rho^{*}=0.4$, one would be hard-
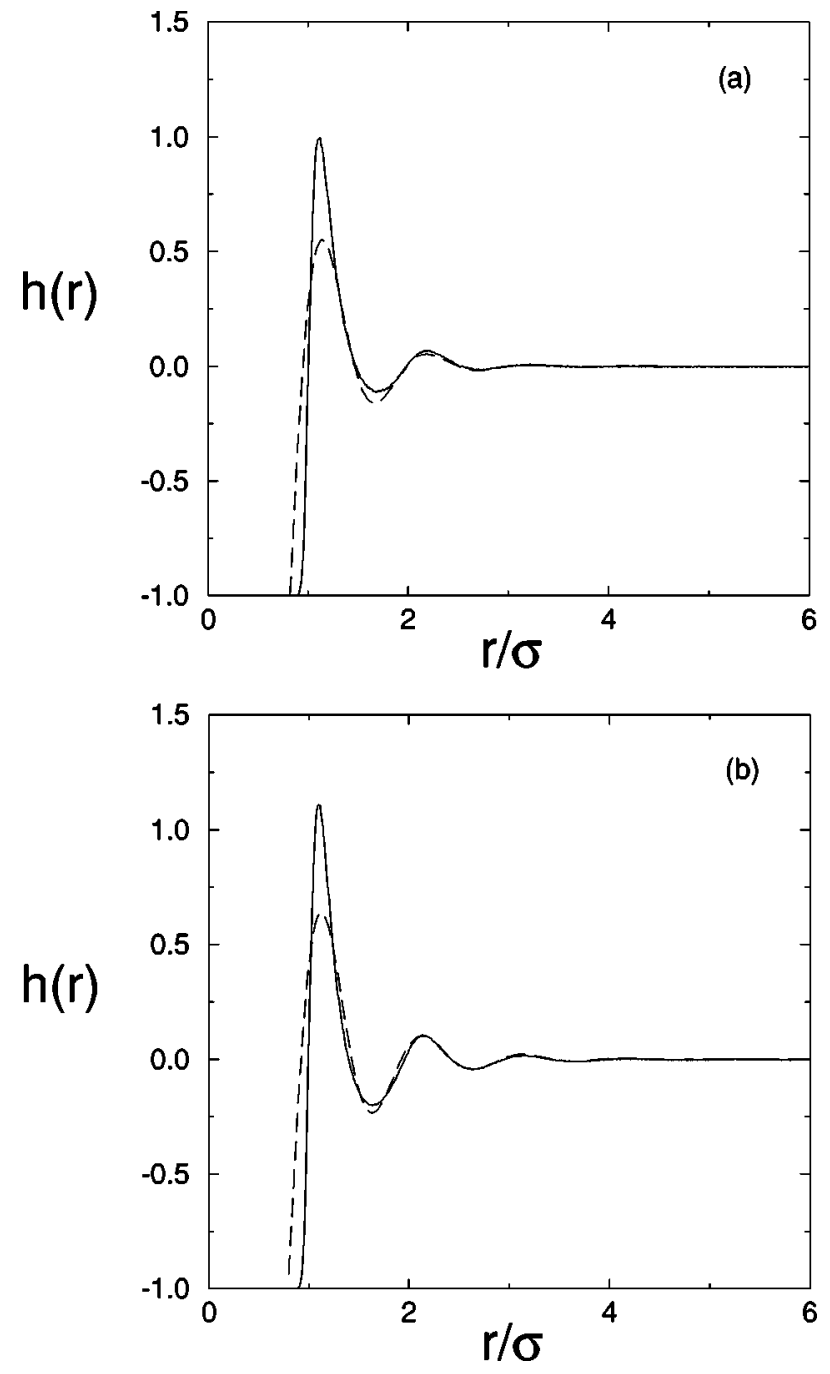

FIG. 5. $h(r)$ for the truncated and shifted LJ fluid at $T^{*}=1.25$. The solid curve is the Monte Carlo result and the dashed curve is the asymptotic result obtained from (23), i.e., retaining the contribution from the single pole on the imaginary axis plus that from the lowest-lying conjugate pair of complex poles. (a) $\rho^{*}=0.4$. The pure imaginary pole has $\alpha_{0} \sigma=0.885$ and the conjugate pair has $\alpha \sigma= \pm 6.105+i 1.718$. Thus $\alpha_{0}<\tilde{\alpha}_{0}$ and the ultimate decay of $r h(r)$ is pure exponential. (b) $\rho^{*}=0.6$. Now the pure imaginary pole has $\alpha_{0} \sigma=1.761$ and the conjugate pair has $\alpha \sigma= \pm 6.273+i 1.241$. Thus $\alpha_{0}>\tilde{\alpha}_{0}$ and the ultimate decay is exponentially damped oscillatory.

pushed to make any definitive statement. Direct observation of crossover from monotonic to damped oscillatory decay of $h(r)$ is extremely difficult in a simulation study, where data are limited by box size and the statistical accuracy may be poor. Our procedure, which inputs data for $h(r)$ at small and intermediate $r$ and provides, via the auxiliary function $\hat{c}(q)$, detailed information about $h(r)$ at large $r$, does appear to be very useful. What its status is as a mathematical extrapolation procedure remains to be investigated.

\section{POLES IN THE HARD-SPHERE FLUID}

The occurrence of both pure imaginary and complex poles in the Lennard-Jones fluid is a consequence of the fact that such potentials have both attractive and repulsive components. For potentials which are purely repulsive and of finite range one expects $r h(r)$ to exhibit exponentially 


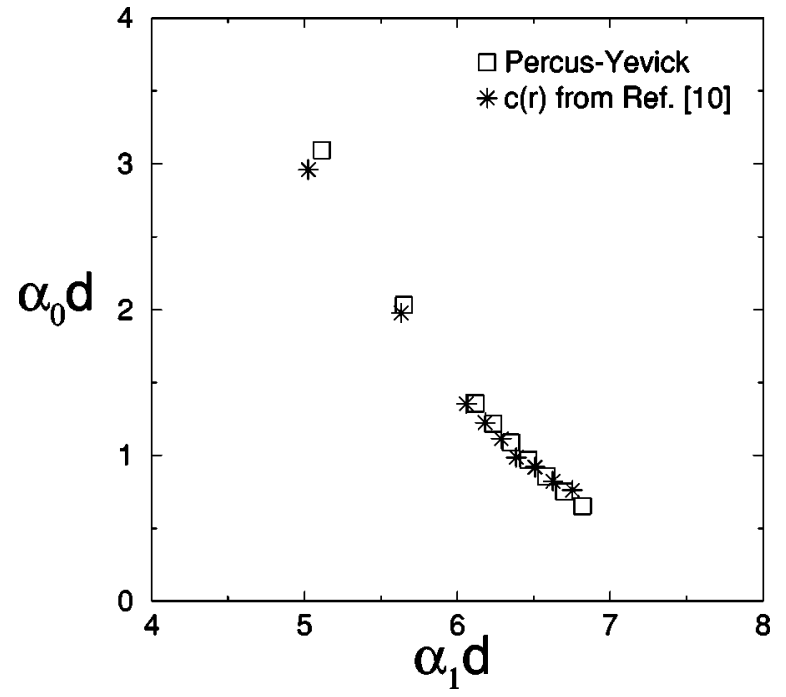

FIG. 6. The imaginary $\left(\alpha_{0}\right)$ and real $\left(\alpha_{1}\right)$ parts of the lowest-lying conjugate pair of poles $\alpha= \pm \alpha_{1}+i \alpha_{0}$ for the hard-sphere fluid, with diameter $d$, for a series of reduced densities $\rho^{*}=\rho d^{3}=0.2$ (top), 0.4, 0.6, 0.65, 0.7, $0.75,0.8,0.85$, and 0.9 (bottom). The squares denote the values from the PY approximation and the asterisks those from the Monte Carlo results for $c(r)$ given in Ref. 17.

damped oscillatory decay for all state points. Of course, the inverse decay length $\alpha_{0}$ and period $2 \pi / \alpha_{1}$ will still depend on the density and temperature. Of special interest is the hard-sphere fluid where the density (or packing fraction $\eta$ $\left.=\pi d^{3} \rho / 6\right)$ is the only relevant variable. $d$ is the hard-sphere diameter. Upon increasing $\eta$ toward the value at freezing ( $\eta \simeq 0.49$ ) one expects, intuitively, that the decay of the oscillations in $h(r)$ will become slower. It is less obvious how the period will vary. There have been surprisingly few studies of the asymptotic decay of $h(r)$ in the hard-sphere fluid. Perry and Throop ${ }^{14}$ calculated the lowest-lying conjugate pair of poles and the corresponding amplitude and phase in (13) from the Percus-Yevick (PY) approximation for the Laplace transform of $r h(r)$. Rosenfeld, ${ }^{15}$ and more recently Leote de Carvalho et al., ${ }^{6}$ investigated several of the PercusYevick poles in the approach to the so-called asymptotic high-density limit $\eta \rightarrow 1$. Henderson and Sabeur ${ }^{16}$ determined the onset of pure oscillatory decay of $h(r)$ for hard spheres, i.e., solutions of (6) with $\alpha_{0}=0$, from a weighted density functional treatment. Here we determine the poles of the hard-sphere fluid using the results of Groot et al., ${ }^{17}$ who extracted $c(r)$ in a careful analysis of Monte Carlo data, and compare these with the results from the PY approximation.

As is well-known, within PY $c(r)$ vanishes for $r>d$ and is a polynomial in $r$ for $r<d$ with coefficients depending on $\eta$. We calculated the lowest-lying conjugate pair of poles using Eqs. (7) and (8) for a range of (fluid) densities. The results for the real and imaginary parts are shown in Fig. 6 and these agree with published results obtained by other methods. ${ }^{6,14}$ In reality the direct correlation function does not vanish identically for $r>d$, rather it has a rapidly decaying tail. Groot et al. ${ }^{17}$ allowed for the possibility of an empirical, damped oscillatory tail in $c(r)$. Their fits (via OZ) to the simulation data for $h(r)$ show that the tail is non-negligible for large packing fractions. However, for all the cases they study the tail is rapidly decaying and $|c(r)|$ is $\leqslant 0.01$ for $r / d \geqslant 2.0$. We used the data for $c(r)$ given in Table II of Ref. 17 along with Eqs. (7) and (8) to obtain the lowest-lying conjugate pair of poles at each density and these results are also shown in Fig. 6. There is rather close agreement between the PY and simulation results across the full range of densities. Given that PY does provide quite a good description of the intermediate range structure in $h(r)$, this level of agreement may not come as a surprise. However, we should remark that since $c(r)$ in Ref. 17 does differ significantly, both inside and outside the hard core, from the PY solution we are inputting two sets of significantly different data into the equations which determine the poles. As expected, the inverse decay length $\alpha_{0}$ decreases rapidly with increasing density until large packings are attained when the decrease becomes much slower. The variation of $\alpha_{1}$ with density is weaker. For $\rho^{*}=0.2$, the period $2 \pi / \alpha_{1}$ is about $1.25 d$ whereas for $\rho^{*}=0.9$ this is $0.94 d$.

\section{THE FW LINE AT HIGH TEMPERATURES}

For a fluid described by a pairwise potential of the Lennard-Jones-type, repulsive forces must always dominate at sufficiently large temperatures and then the structure of the fluid must resemble that of a fluid in which the potential is hard-sphere like, for which the decay of $h(r)$ is always damped oscillatory and no crossover exists. Earlier studies investigated the FW line for temperatures up to about twice the critical temperature 2,5 where the effects of attractive forces are still very strong and one is far from approaching hard-sphere like behavior. Here we address the issue of what happens to the FW line for very high $T^{*}$.

Rather than extending the simulation study of Sec. III, which would be extremely laborious, we have used the RPA to map out the FW line in the $\left(T^{*}, \rho^{*}\right)$ plane for the LJ fluid with potential (22). The RPA assumes that the direct correlation function can be approximated by

$$
c_{\text {rpa }}(r)=c_{h s}(r)-\beta \phi_{\text {att }}(r),
$$

where $c_{h s}(r)$ is the PY hard-sphere direct correlation function at density $\rho^{*}$ and the attractive potential is chosen to be

$$
\begin{aligned}
\phi_{\mathrm{att}}(r) & =-\epsilon \quad r \leqslant r_{\min } \equiv 2^{1 / 6} \sigma \\
& =\phi_{\mathrm{LJ} t s}(r) \quad r>r_{\min } .
\end{aligned}
$$

For simplicity, the hard-sphere diameter $d$ is set equal to $\sigma$. Other choices of the attractive potential inside the core are possible and it is well-known that $h(r)$ calculated from the RPA closure is not necessarily zero inside the repulsive core. Nevertheless, the pole structure arising from the RPA does reproduce the key features of more sophisticated closure approximations. $^{2,5}$ Once again the lowest-lying poles were calculated using Eqs. (7) and (8) and the FW line was determined as in Sec. III. The results are shown in Figs. 7 and 8. At temperatures in the range $1.0 \leqslant T^{*} \leqslant 1.8$, the RPA FW line has a similar (near linear) shape to the line obtained in simulation (and in the HMSA). The RPA line intersects the (simulation) coexistence curve at a slightly higher temperature, $T^{*}=1.015$, than our simulation estimate $T^{*}=0.95$. For higher temperatures, the FW line increases rapidly with de- 


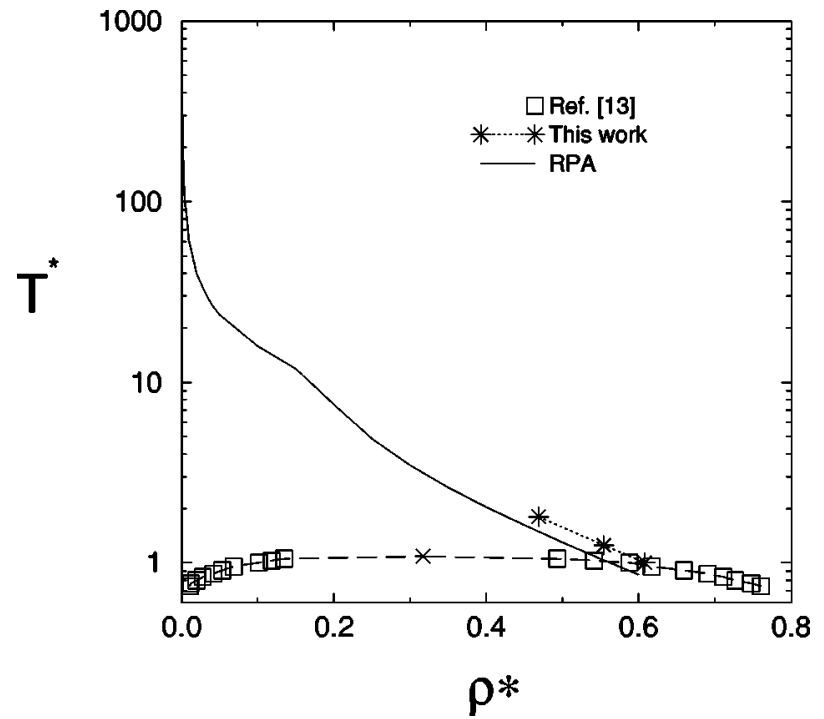

FIG. 7. FW line (solid curve) for the truncated and shifted LJ fluid at high temperatures as obtained from the RPA. The asterisks joined by dots denote our Monte Carlo results for the FW line. The dashed line joining squares denotes simulation results (Ref. 13) for the liquid-gas coexistence curve and the cross marks the critical point-see Fig. 4.

creasing $\rho^{*}$. Crossover from monotonic to damped oscillatory decay of $h(r)$ occurs at the critical density $\rho^{*}=\rho_{c}^{*}$ when $T^{*} \simeq 3.171$, i.e., $T / T_{c} \simeq 2.923$. At a fairly low density, $\rho^{*}=0.03\left(\sim 0.1 \rho_{c}^{*}\right)$, crossover does not occur until $T^{*}$ $\simeq 32$. One deduces that the attractive interactions, which give rise to the pure imaginary pole, continue to manifest themselves up to very high temperatures when the density is low. Figure 7 shows that the FW line approaches the temperature axis asymptotically, as expected, and it is clear that

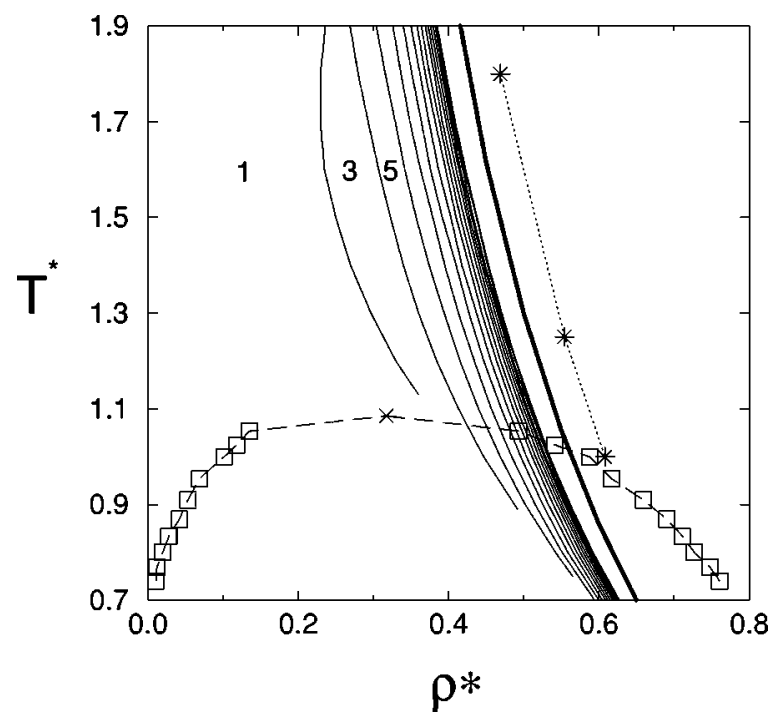

FIG. 8. Boundaries separating regions in the temperature-density plane with $n$ and $n+2$ zeros of $h(r)$ for the truncated and shifted LJ fluid. The solid curves are RPA results for the loci of the boundaries between regions with $1,3,5, \ldots$ zeros. On these boundaries, the last, $(n+1)$ th zero is a minimum of $h(r)$. For large $n$ the boundaries crowd together and as $n \rightarrow \infty$ they approach the FW line (bold curve). The asterisks joined by dots denote our Monte Carlo results for the FW line. The dashed line joining squares denotes simulation results (Ref. 13) for the liquid-gas coexistence curve and the crossmarks the critical point. in a very low density fluid the ultimate asymptotic decay of $h(r)$ will not resemble that of hard spheres until extremely high temperatures.

\section{THE NUMBER OF ZEROS OF $h(r)$}

On the oscillatory side of the FW line, $h(r)$ has an infinite number of zeros since the dominant decay is given by Eq. (13). On the monotonic side oscillations do still occur. It is only the ultimate decay of $r h(r)$ which is pure exponential (10) and the lowest-lying complex poles make increasingly important contributions as the FW line is approached. Rewriting the two-pole approximation (23) as

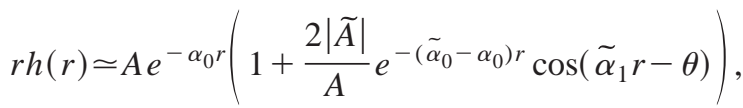

we see that, provided the ratio of amplitudes is sufficiently large, the second (oscillatory) term will remain comparable with the first at increasingly large distances as $\tilde{\alpha}_{0} \rightarrow \alpha_{0}$. In turn, this implies an increasing number of zeros of $h(r)$ will be exposed on approaching the FW line and one can envisage boundaries in the $(T, \rho)$ plane separating a region where $h(r)$ has $n$ zeroes from one where it has $n+2$ zeros. On the boundary there will be $n+1$ zeros with the $(n+1)$ th being an extremum. As $n$ increases, these boundaries should crowd closer together and converge to the FW line, which corresponds to $n=\infty$.

We have calculated the loci of these boundaries for the truncated and shifted LJ fluid using the RPA, i.e., Eqs. (24) and (25). Our calculations employed the two-pole approximation (23) with the poles, amplitudes, and phase determined as previously. As argued in Sec. III B, this approximation yields an accurate description of the intermediate range behavior of $h(r)$ provided the next lowest-lying conjugate pair of poles has an imaginary part $\gg \tilde{\alpha}_{0}$. We checked, by computing the full $h(r)$ from the OZ relation, that (23) counts correctly the first few zeros. Upon increasing $\rho^{*}$ at fixed $T^{*}$, one passes from a region where $h(r)$ has one zero to one where it has three zeros and then to one with five zeros and so forth. The boundaries separating these regions are shown in Fig. 8. Pronounced crowding of the boundaries has already occurred for $n \sim 20$, at densities which are still well below that of the FW line. Note that except exactly on a boundary, $h(r)$ always has an odd number of zeros. We can understand this as follows. In the limit $\rho \rightarrow 0, h(r) \sim \exp$ $(-\beta \phi(r))-1$ which has one zero. The presence of the cosine in the two-pole approximation (26) implies that the number of zeros increases by two upon crossing the first boundary. On the boundary there are two zeros, the second corresponding to a minimum of $h(r)$. The sequence continues so that on the monotonic side of the FW line $h(r)$ always decays to zero from above as $r \rightarrow \infty$. The amplitude $A$ in (26) must be positive. These conclusions have been confirmed within the RPA. Our simulation results also appear to be consistent with the observation that $h(r)$ should decay to zero from above, although it is difficult to check this directly when $n$ $>7$. Although we are not aware of any rigorous proof that in 
the region of ultimate monotonic decay $h(r)$ should decay from above, such a result is in keeping with Widom's proof that $\int d \mathbf{r}(g(r) \exp (\beta \phi(r))-1) \geqslant 0 .{ }^{8}$

\section{CONCLUDING REMARKS}

We have shown that computer simulation results can be used to determine the longest range asymptotic decay of $h(r)$ for fluids exhibiting finite-range interatomic potentials. By calculating $c(r)$ from the simulation results we were able to determine the lowest-lying poles of $\hat{h}(q)$, which enabled us to calculate a portion of the crossover (FW) line for the truncated and shifted Lennard-Jones fluid. The FW line lies very close to the previous HMSA results, implying that the latter provides an accurate description of the lowest-lying poles. In order to calculate the poles we had to make certain assumptions about the asymptotic decay of $c(r)$, i.e. that this function decays sufficiently rapidly to employ Eqs. (7) and (8). Although these assumptions appear to be justified in the present case, future work should reexamine them. This will become increasingly important as the potential cutoff $R_{c}$ increases. ${ }^{5,18}$ For fluids with true power-law potentials or with exponentially decaying (Yukawa) pair potentials, alternative procedures are required to determine the poles $^{5-7}$ and it remains a challenge to implement these within simulation.

Although our present technique does not allow us to calculate reliably the higher-lying poles, the success of the twopole asymptotic approximation in describing the intermediate range features of $h(r)$ implies the next lowest-lying pole is well-removed from those we do determine and that contributions to $h(r)$ from other types of singularity do not have a major influence. Were such an observation to be valid, generally this would lead to important simplifications in developing a fundamental understanding of correlation functions.

Our results for the asymptotic decay of $h(r)$ in the hardsphere fluid extend to reduced densities $\rho d^{3} \equiv \rho^{*}=0.9$. It is interesting that the inverse decay length $\alpha_{0}$ (see Fig. 6) obtained from simulation results decreases less rapidly at high $\rho^{*}$ than in the PY approximation. Further simulation results for densities up to freezing $\left(\rho^{*} \simeq 0.94\right)$ could confirm this trend and test the reliability of density functional treatments in the high-density region. Note that PY approximation for hard spheres (at densities well-beyond freezing) does not predict an instability to pure oscillatory solutions whereas certain density functional treatments for $c(r)$ do. ${ }^{16}$
The last two sections of our paper dealt with the FW line at high temperatures and with the location of boundaries separating regions in the $(T, \rho)$ plane where $h(r)$ has a different numbers of zeros. To the best of our knowledge, neither of these topics has been considered previously. Our results are based on the RPA which, although crude, should capture most of the key features of the decay of $h(r)$. In particular we would expect the overall shape of the FW line in Fig. 7 and the form and clustering of the boundaries in Fig. 8 to be given correctly by the RPA. This could, of course, be tested by employing more sophisticated integral equation theories or, indeed, simulations.

\section{ACKNOWLEDGMENTS}

The calculation described in Sec. VI was suggested by Ben Widom. We are grateful that he can recall his ideas from 30 years ago and explain them with such clarity. This work was made possible by financial support under EPSRC Grant No. GR/L89013.

${ }^{1}$ M. E. Fisher and B. Widom, J. Chem. Phys. 50, 3756 (1969).

${ }^{2}$ R. Evans, J. R. Henderson, D. C. Hoyle, A. O. Parry, and Z. A. Sabeur, Mol. Phys. 80, 755 (1993).

${ }^{3}$ R. Evans, R. J. F. Leote de Carvalho, J. R. Henderson, and D. C. Hoyle, J. Chem. Phys. 100, 591 (1994).

${ }^{4}$ J. R. Henderson, Phys. Rev. E 50, 4836 (1994).

${ }^{5}$ R. J. F. Leote de Carvalho, R. Evans, D. C. Hoyle, and J. R. Henderson, J. Phys.: Condens. Matter 6, 9275 (1994).

${ }^{6}$ R. J. F. Leote de Carvalho, R. Evans, and Y. Rosenfeld, Phys. Rev. E 59, 1435 (1999)

${ }^{7}$ R. J. F. Leote de Carvalho and R. Evans, Mol. Phys. 83, 619 (1994); 92, 211 (1997).

${ }^{8}$ B. Widom, J. Chem. Phys. 41, 74 (1964).

${ }^{9}$ J. E. Enderby, T. Gaskell, and N. H. March, Proc. Phys. Soc. London 85, 217 (1965).

${ }^{10} \mathrm{We}$ expect an infinite number of conjugate pairs of complex poles but, provided the moments of $c(r)$ satisfy certain conditions, there should be a single pure imaginary pole at any given state point-see Ref. 5. In our present simulations we do find only one such pole.

${ }^{11}$ G. Zerah and J. P. Hansen, J. Chem. Phys. 84, 2336 (1986).

${ }^{12}$ In our calculations, we used the Fourier transform method that is described in Computer Simulation of Liquids, edited by M. P. Allen and D. J. Tildesley (Oxford University Press, Oxford, 1994).

${ }^{13}$ B. Smit, J. Chem. Phys. 96, 8639 (1992).

${ }^{14}$ P. Perry and G. J. Throop, J. Chem. Phys. 57, 1827 (1972).

${ }^{15}$ Y. Rosenfeld, Phys. Rev. A 33, 2025 (1986).

${ }^{16}$ J. R. Henderson and Z. A. Sabeur, J. Chem. Phys. 97, 6750 (1992); Mol. Phys. 82, 765 (1994).

${ }^{17}$ R. D. Groot, J. P. van der Eerden, and N. M. Faber, J. Chem. Phys. 87, 2263 (1987).

${ }^{18}$ C. Vega, L. F. Rull, and S. Lago, Phys. Rev. E 51, 3146 (1995). 